\title{
REVIEW OF WAVELET THEORY AND ITS APPLICATION TOIMAGE DATA COMPRESSION
}

\author{
OTHMAN OMRAN KHALIFA \\ Electrical \& Computer Department, Kulliyyah of Engineering, International Islamic \\ University Malaysia, Jalan Gombak, 53100, Kuala Lumpur, Malaysia \\ Fax: +6032056 4853, Email: khalifa@iiu.edu.my
}

\begin{abstract}
The fast development of computing multimedia has led to the demand of using digital images. The manipulation, storage and transmission of these images in their raw form is very expensive, it significantly slows the transmission and makes storage costly. In this paper, a brief review of wavelet transform theory is given using filters as examples to show the related multiresolution analysis. Advantages over Fourier transform is investigated and several results are derived. The pyramid algorithm is also presented and some features of wavelets in image data compression are given. A modified version of Lind Boze and Gray (LBG) algorithm using Partial Search Partial Distortion (PSPD) is presented for coding the wavelet coefficients to speed up the codebook generation and the search required for nearest neighbour codevector of input image. The proposed scheme can save $70-80 \%$ of the Vector Quantization (VQ) encoding time as compared to fully search VQ and reduced arithmetic complexity with less sacrificing performance.
\end{abstract}

Key Words: Image compression, Wavelets transform, Vector qQuantization.

\section{INTRODUCTION}

Wavelet Transform [1-3] has emerged as a powerful mathematical tool in many areas of science and engineering specifically for data compression [4,5]. The wavelet transform was first introduced in the context of a mathematical transform in 1984 by A. Grosman and J. Morlet. Although Haar constructed the first wavelet system in 1910. Daubechies brought a big breakthrough in 1988 [6], her work immediately stimulated a rapid development in the theory and applications of wavelet analysis. In 1989, Mallat presented the theory of multiresolution [3], with this technique; the wavelet system can be constructed with desired properties. Wavelets has provided a promising vehicle for image processing applications, because of its flexibility in representing images and its ability to take into account Human Visual System characteristic. It is mainly used to decorrelate the image data, so the resulting coefficients can be efficiently coded. It also has good energy compaction capabilities, which results in a high compression ratio. It can be viewed as a special case of multi-rate filter bank with dyadic tree decomposition.

Wavelets were developed independently in the fields of mathematics, physics, electrical engineering and seismic-geology. Interchanges between these fields during the 
last ten years have led to many wavelet applications, such as image compression, denoising, human vision, radar, and the list could be continue. Also, it is being used in many areas of science and engineering such as: signal processing, fractal analysis [7][8], numerical analysis [9], statistics [10], and astronomy [11]. Recently, wavelets were determined to be the best way to compress a huge library of fingerprints [12].

The advantages of using the Discrete Wavelet Transform (DWT) over the Discrete Cosine Transform (DCT) lies in the fact that the DWT projects high details of image components onto shorter basis function, with higher resolution, where lower detail components are projected onto larger basis functions, which corresponds to narrower subbands $[8,13,14]$. In addition, the wavelet transform compression provides a superior image quality at a low bit rate, since it is free from blocking effects. Also, it has a link with digital filters and can handle non-stationary signals. However, there are still significant amounts of redundancies within the subbands.

The goal of most wavelet researchers is to create a set of basis functions and transforms that will give an informative, efficient, and useful description of a function or signal. If the signal is represented as a function of time, wavelets provide efficient localisation in both time and frequency $[3,15]$.

Since the wavelet basis functions have short support for high frequencies and long support for low frequencies, smooth area of an image may be represented with very few bits. It is known that most of the energy is concentrated in low frequency information, and for the remaining high frequency components of the image, most energy is spatially concentrated around edges. High frequency details are added where they are needed. Indeed, before the introduction of wavelets, a number of closely related coding works have been extensively studied in the image coding community, including pyramid coding [16], where the coarse version is derived from the original image by filtering. From this coarse version, the original image can be predicted and the prediction error can be calculated. If the prediction error is small it can be well compressed. The process can be iterated on the coarse version. A perfect reconstruction can be achieved if the compression of difference signals is lossless by simply predicting the original image and adding back the predicted image and the difference, the compression rate depends on how well the original image can be predicted from the filtered and down sampled image. They split up the input image into frequency bands and then code each subband using coder bit rate method to the statistics of the band.

Initial efforts in using wavelet transform in compression research concentrated on the hope of more efficient compaction of energy into a few number of low frequencies. This generated some wavelet - based coding algorithms [7,17] which were designed to exploit the energy compaction properties of the wavelet transform by applying scalar or vector quantizers for the statistical of each frequency band of wavelet coefficients.

\section{WAVELET TRANSFORM HISTORY ADVENT}

The basic idea of applying a wavelet transform for image compression is that of successive approximation of an input image signal at reduced resolution together with the added detail signal at that resolution. This wavelet representation provides a 
multiresolution and multi-frequency expression of nonstationary images with localisation in both time and frequency domain. It is a tool which breaks up data into different frequency components or subbands and then studies each component with a resolution that matched to its scale. There is continuous wavelet transform (CWT) and discrete wavelet transform (DWT).

Definition: 2.1 The continuous wavelet transform (CWT) of a function $f(x) \in L^{2}(R)$ with respect to $\varphi(x) \in L^{2}(R)$ is

$$
C W T(f)(a, b)=|a|^{j / 2} \int_{-\infty}^{\infty} f(x) \varphi(a(x-b)) d x
$$

where, $a, b, \in R, \quad a \neq 0$ and $j$ is a resolution number.

Definition 2.2 The discreet wavelet transform (DWT) of a function $f(x) \in L^{2}(R)$ with respect to $\varphi(x) \in L^{2}(R)$ is

$$
D W T(f(j, k))=\left|a_{0}\right|^{j / 2} \int_{-\infty}^{\infty} f(x) \varphi\left(a_{0}{ }^{j} x-k b_{0}\right) d x
$$

where, $a_{0}, b_{0}, \in R, \quad a_{0} \neq 0, \quad j, k \in z$

For the discrete wavelet transform $a_{0}$ and $b_{0}$ are usually set to 2 and 1 . In such case, the DWT becomes:

$$
D W T(f(j, k))=2^{j / 2} \int_{-\infty}^{\infty} f(x) \varphi\left(2^{j} x-k\right) d x
$$

\section{WAVELET ANALYSIS AND COMPARISON WITH FOURIER TRANSFORM}

A wave is usually defined, as an oscillating function of time, such as a sinusoid. Fourier analysis expands signal or functions in terms of sinusoids, which has proven to be extremely valuable. Wavelets are mathematical functions that cut up data into different frequency components. It has advantages over traditional Fourier methods in analysing physical situations where the signal contains discontinuities and sharp spikes. By comparing wavelets with sine waves, which are the basis of Fourier analysis, sinusoids do not have limited duration that is, they extend from minus to plus infinity. And where sinusoids are smooth and predictable, wavelets tends to be irregular and asymmetric. In 1907 Joseph Fourier showed that any $2 \pi$ periodic function could be expressed in terms of its complex exponential, which enables breaking up a signal into sine waves of various frequencies. For stationary signal, the Fourier transform can be defined as: 


$$
X(\omega)=\int_{-\infty}^{\infty} x(t) e^{-j \omega t} d t
$$

Similarly, wavelet analysis is the breaking up a signal into shifted and scaled versions of the original wavelet as follows:

$$
\psi_{a, b}(t)=a^{-1 / 2} \psi\left(\frac{t-b}{a}\right)
$$

where is $a$ and $b$ are scale and shift parameters respectively.

In Fourier transform domain, we completely lose information about the localisation of the features of an image, quantisation error on one coefficient can affect the quality of the entire picture. The wavelet representation explicitly contains position information: the index $a$ of wavelet $\psi_{a, b}$ indicates by how much the mother wavelet needs to be shifted to obtain one of the basis functions. The size of the region affected depends on how well the mother wavelet is localised. Also, all wavelets tend to zero at infinity, which is already better than the Fourier basis function. Furthermore, wavelets can be made to tend to zero as fast as possible. Figure 1 shows the original signal, Fig. 2 shows the reconstructed signal via the Fourier transform while Fig. 3 shows the reconstructed signal using wavelet transform.

In a Fourier analysis a single point in the Fourier domain contains information from everywhere in the image. It specifies the amount or strength of that particular spatial frequency that exist in the image. In the image domain, the existence of a strong high frequency component means that there are edges in the image, it shows how their orientation relates to the orientation of the spatial frequency, but the frequency domain result does not tell us where they exist in the image.

In fact one could not even know if the edge information is constrained to one particular area of the image or if it is dispersed over the entire image as a texture pattern. Technically, it says that the Fourier basis functions have infinite support. Practically, this fact has important ramifications. In other words, image compression using the Fourier transform is independent of the image context. This is in many cases a disadvantage. Often what is needed in image compression is a scheme that retains details where required and compresses non-significant areas of the image. Wavelets, on the other hand, have compact or finite support and it enables different parts of the image to be represented at different resolution.

Fourier transform has been successful in a wide range of applications, however, it is suitable only if the signal consists of a few stationary components. Also, the amplitude spectrum does not provide any idea of how the frequency components evolve with time. This drawback can be overcome by applying a time window on the data and then taking the Fourier transform. This is known as short-time Fourier transform (STFT), which can be calculated as 


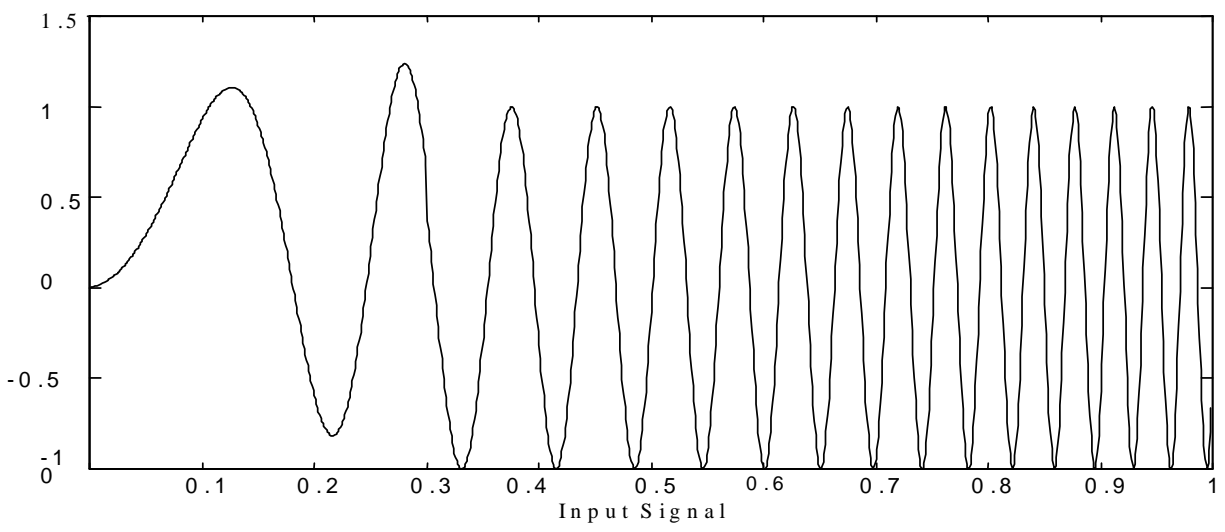

Fig. 1: Input signal (one dimension example).

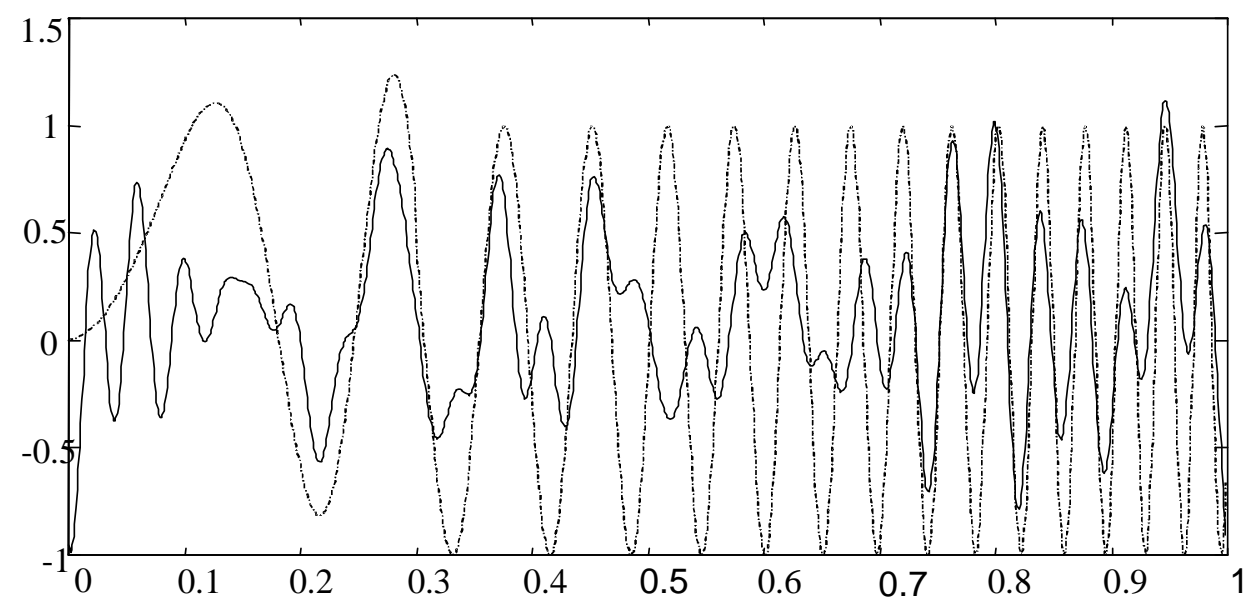

Fig. 2: Fourier approximation

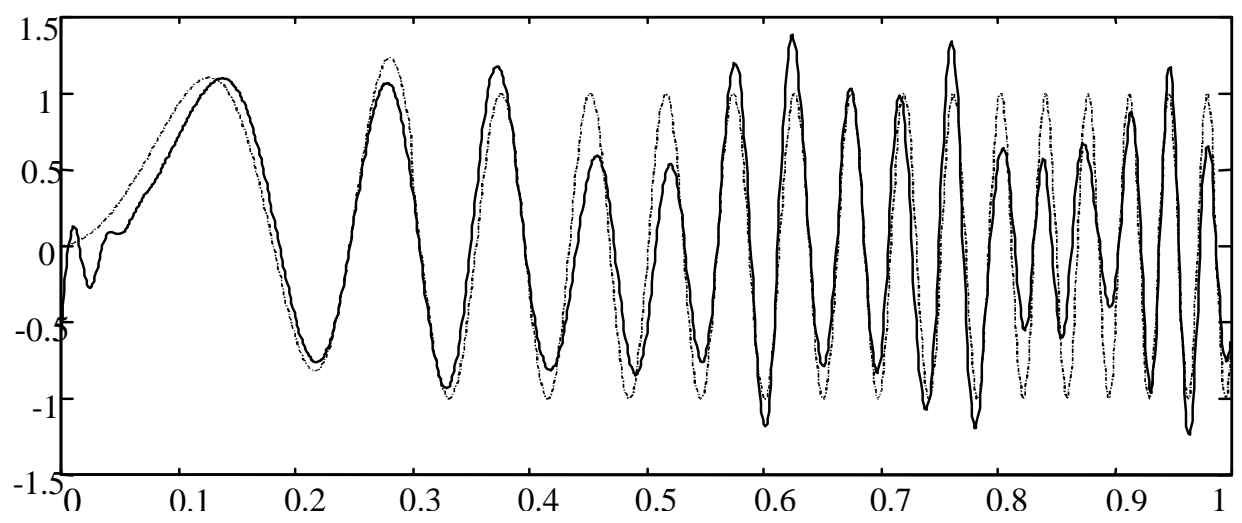

Fig. 3: Wavelet approximation. 


$$
\operatorname{STFT}_{x}(\omega, b)=\int_{-\infty}^{\infty} x(t) g^{*}(t-b) e^{-j \omega t} d t
$$

where $x(t)$ is the input signal and the window $g(t-b)$ is a time-localised function is shifted over the time axis to calculate the transform at several time locations $b$. One of the commonly used is the Gaussian time window with a corresponding Gaussian frequency window. This transform is called the Gabor transform.

The STFT has two major drawbacks. First, both the time and frequency windows are fixed. Hence, STFT cannot adapt to the signal statistics. A short time window will be able to detect the high frequency components, but will provide poor response to low frequency components and vice versa. Secondly, it is desirable to have a window function that has some decay in the time domain as well as in the frequency domain. Wavelet transform overcomes both of these drawbacks.

It can be viewed as dilated and translated versions of the mother wavelet $\psi(t)$. The arguments $a$ and $b$ denote scale and location parameters respectively. Dilation corresponds to the change of frequencies and translation refers to localisation of time or position. The tiling of the time-frequency plane for both STFT and WT is shown in Fig. 4. It is noted that the time and frequency windows are fixed for STFT, once the width of the window $W(t, T)$ is chosen, this tiling is fixed. However, it would be desirable to study the slowly varying properties of the signal (low frequencies) over a longer time span and vice versa for high frequencies. But this is provided by employing DWT as analysing functions. For higher scale, the time window narrows and for lower scale, it widens. This type adaptation is very useful in most practical situations. Since, the high frequency components are normally short lived and hence need a narrow time window to improve the time resolution. On the other hand, low frequency components change slowly over time, thus requiring a wider time window to detect the slow changes.

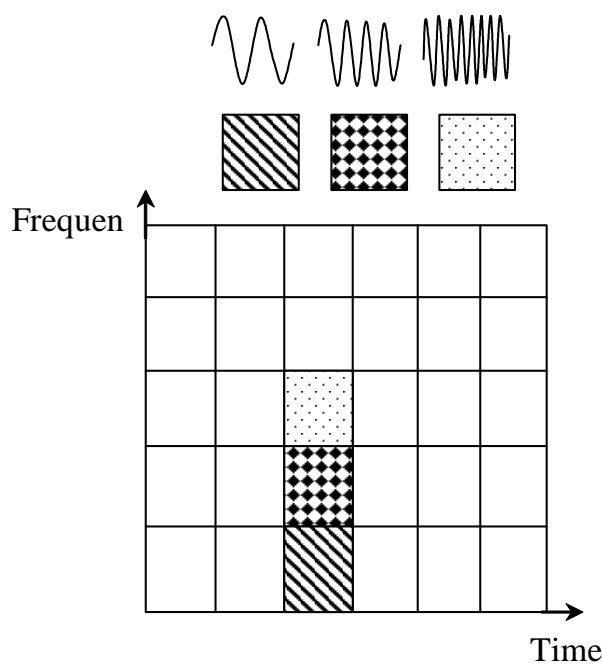

(a)

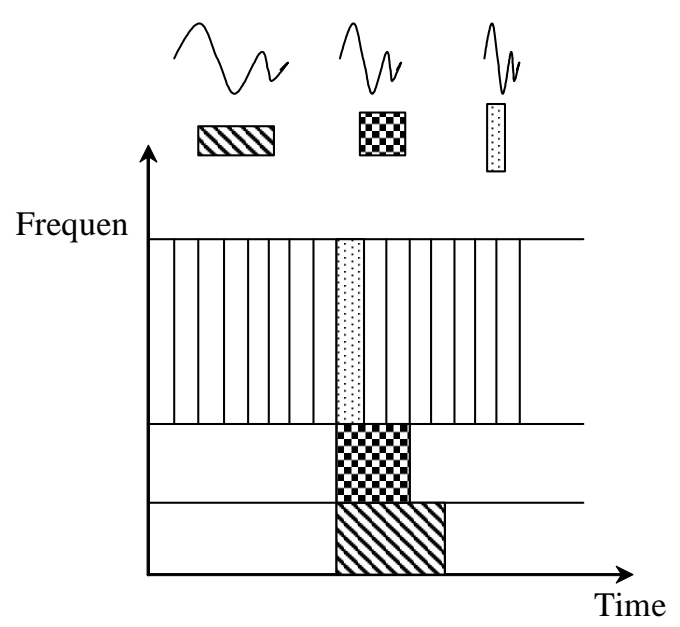

(b)

Fig. 4: Time-frequency plane showing resolution cells

a) STFT, b) WT 


\section{THE DISCRETE WAVELET TRANSFORM}

The application of wavelet transform in signal and image compression has attracted a great deal of attention. It is known that it generates a multiresolution representation of an image. There are several subimages or subbands that might be encoded more perfectly than the original image. The wavelet transform technique breaks the image information into various frequency bands and encodes each subband using suitable coding system.

Consequently, different coding approaches or different bit rates could be assigned to every subimage. The separate coding of different subbands provides some desirable features. First, by allocating the available bits for encoding among the subbands and using an appropriate quantizer for each of them, the encoding process can be tailored to the statistics of each subband. Second, spectral shaping of the quantisation noise is possible. This feature can be used to take advantage of the noise perception of the human auditory system for speech or the human visual for images. Third, the subband decomposition of the signal or image leads naturally to multiresolution decomposition. This is useful for progressive transmission of images in which an increasingly higher resolution and quality image can be reconstructed by the decoder. To get high compression ratio, one can not code the whole information of an image. Only the significant information of an object is needed to reconstruct the image with less distortion or degradation.

A more sophisticated wavelet can also provide more energy compaction than Haar wavelet. Daubechies et al [18] has shown that a wavelet ability measure is to provide compaction by the number of vanishing moments it possesses. More vanishing moments imply more compaction in smooth regions. The Haar wavelet has only one vanishing moment. Therefore, it does not possess a very strong compaction ability.

Daubechies has generated a family of wavelets parameterised by the number $\mathrm{N}$ of vanishing moments [18]. This family has $2 \mathrm{~N}$ non-zero coefficients. These wavelets have problems because of the asymmetry that it introduces which result in subjective displeasing distortions when errors are introduced into the decomposition coefficients. She also designed another family of wavelets called the coiflet. It has vanishing moments for both the wavelets and the scale function. These filters are very close to symmetric where this wavelet is increased filters length: for L vanishing moments, the filters have 3L non-zero coefficients. Fig. 5 and Fig. 6 show the $\mathrm{L}=12,8$ coiflet and Daubechies Coefficients respectively. 


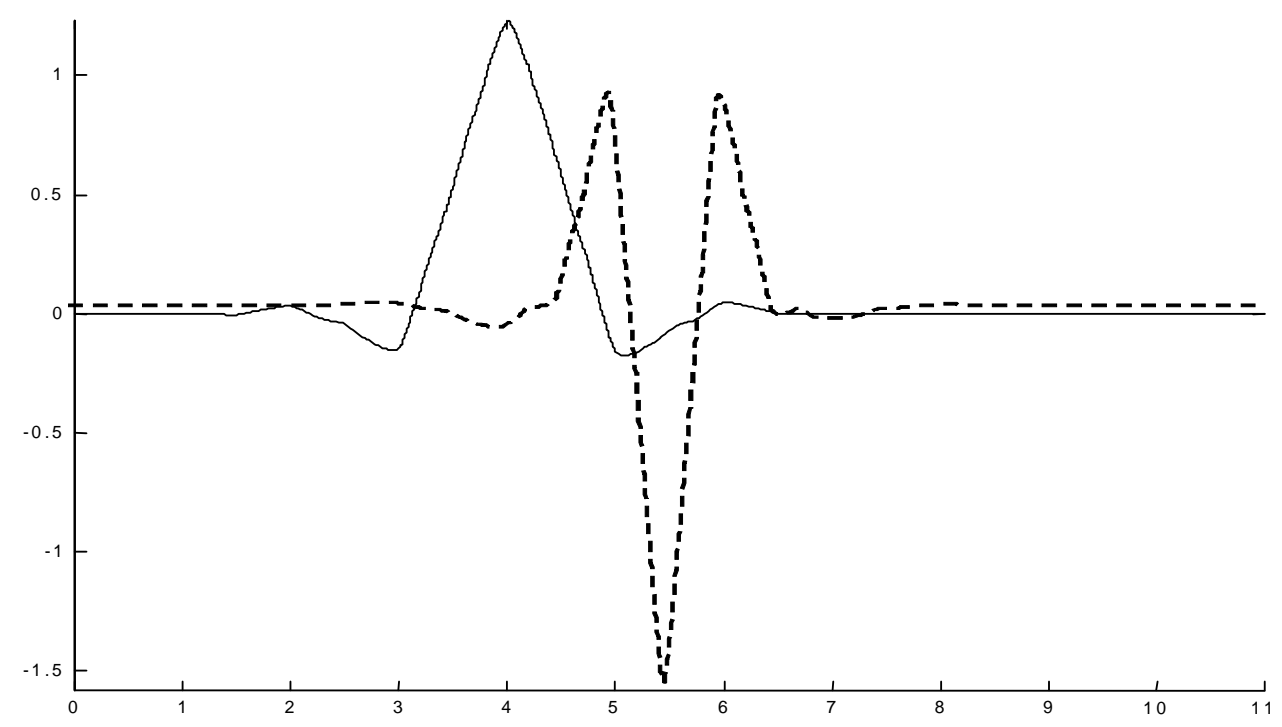

Fig. 5: Coiflet Coefficients, L=12 (a) Scaling function (Bold line) (b) Wavelet (dash line).

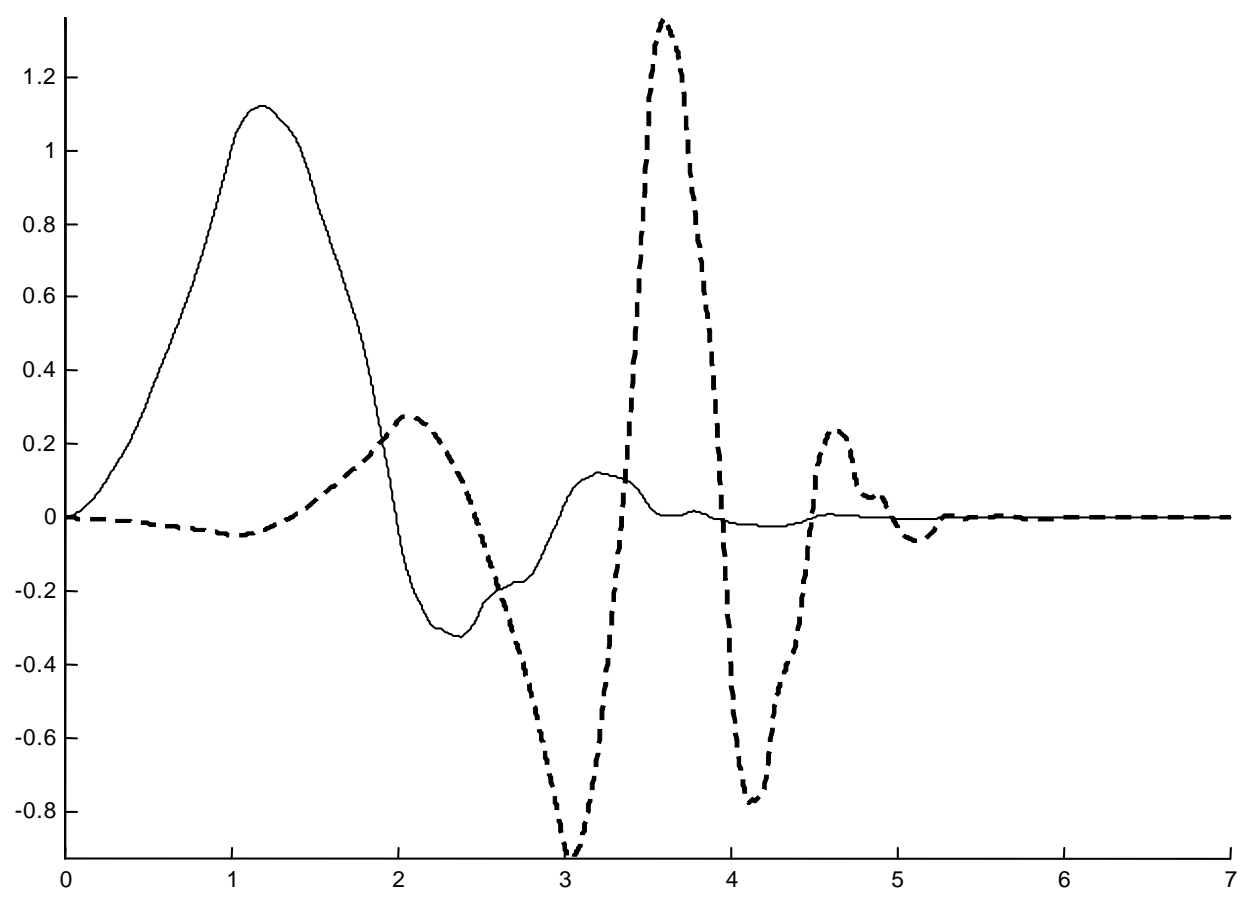

Fig. 6: Daubechies Coefficients, $\mathrm{L}=8$ (a) Scaling function (Bold line), (b) Wavelet (dash line). 


\section{DESIGN OF WAVELET FILTERS}

This section describes the design procedure for two popular wavelets, namely Daubechies and Coiflet family. A comparative study to determine the role of different characteristics of the wavelet filters $e . g$. regularity, perfect reconstruction linear phase, filter length, and coding gain, to the performance of wavelet compression proposals can be found in [23].

The choice of filter bank in wavelet compression is an important issue that can affect image fidelity as well as system design. The efficiency of any wavelet transform coder essentially depends on the energy compaction by the DWT. There is considerable flexibility in designing the filter bank where the transforms can be implemented.

During the appropriate design the following characteristics are desirable:

- Perfect reconstruction or the ability to exactly obtain the original input.

- Orthonormal or biorthogonal bases.

- Reasonable spatial and frequency resolution.

The two-channel filter bank has four filters involved: two are analysis filters and two are synthesis filters. The system is said to be a biorthogonal filterbank if $R\left(e^{j \omega}\right) E\left(e^{i \omega}\right)=I$ for all $\omega$. This implies perfect reconstruction, i.e., $\hat{x}(n)=x(n)$. The coder is orthonormal or paraunitary if $E\left(e^{j \omega}\right)$ is unitary for all $\omega$. In this case biorthogonality is achieved by setting $R\left(e^{j \omega}\right)=E^{+}\left(e^{j \omega}\right)$.

The DWT can be easily implemented using finite impulse response filter banks $[17,19,20]$. Two-dimensional (2-D) of DWT can be obtained by a separable decomposition in the horizontal and vertical directions [3,21]. A pair of appropriately designed Quadrature Mirror Filters (QMFs) can efficiently implement the forward and inverse wavelet transforms. Therefore, it can be viewed as a form of subband coding. The Quadrature Mirror Filter (QMF) pair consists of a low pass filter $G=G(h(n))$, and $H=H(h(n)), n \in z$, where $h(n), g(n)$ are Daubeche's 6-tap filter. The impulse response $H$, and $G$ are mirror images, and related by:

$$
g_{n}=(-1)^{1-n} h_{1-n}
$$

The impulse response of the forward and inverse transform QMFs - denoted $(\widehat{H}, \widehat{G})$ and $(H, G)$ respectively are related by:

$$
\begin{aligned}
& g_{n}=\widehat{g}_{-n} \\
& h_{n}=\widehat{h}_{-n}
\end{aligned}
$$

Each pair consists of a lowpass filter $(\mathrm{H})$ and a highpass filter $(\mathrm{G})$ which divide or split the input signal/image into two components. 
The choice of filter bank in wavelet compression is an important issue that affects image quality as well as system design. Figure 7 illustrates a single 2-D forward wavelet transform of an image, which is implemented by two separate 1-D transforms. The image $f(x, y)$ is first filtered along the $\mathrm{x}$-dimension, then down sampled by 2 , then filtered along y-dimension and down sampled by 2 , resulting in four subimage $f_{1-1}(x, y)$, $f_{1-2}(x, y), f_{1-3}(x, y)$ and $f_{1-4}(x, y)$. The resulting are the main signal $f_{1-1}(x, y)$ which is the low frequencies band and three detail signals. The process is repeated on the low band to generate the next level of the decomposition.

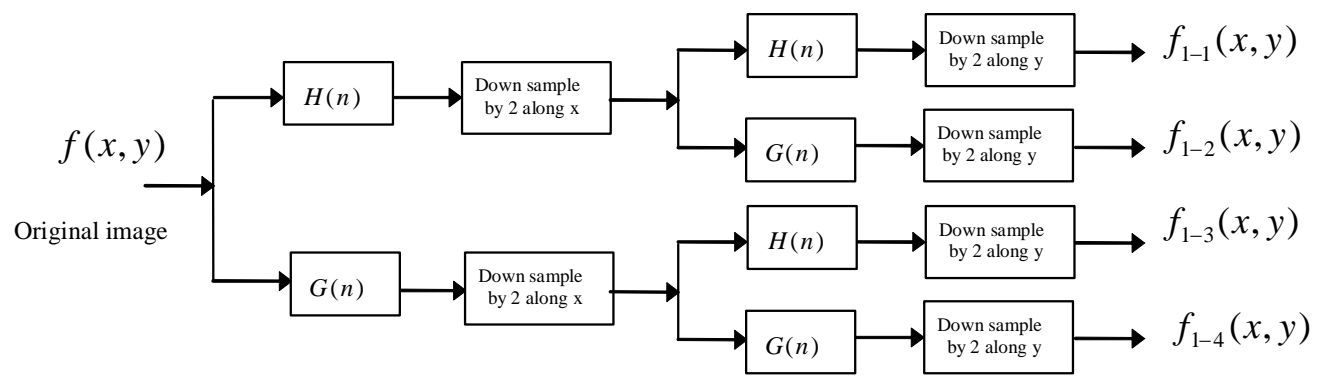

Figure (7) Block diagram of 2-D forward Wavelet Transform

\section{WAVELET REPRESENTATION}

There are many types of wavelets with different associated representations in the context of image compression or coding. We consider only separable 2-D wavelets, which are implemented by corresponding 1-D wavelets and scaling functions. In this paper, the biorthogonal wavelet has been used because of the linear phase and compactness properties. Most of the wavelet applications are in image coding and image processing [3,22,23].

However, the pyramid wavelet transform that is going to use is not suitable for compression of texture-dominant images due to its significant middle frequency components. This can be demonstrated if the first wavelet (pyramid) is taken of that image, it can note the energy compaction is not concentrated in LL subband as the natural images as shown in Table 1. In fact there are many types of wavelet such as full wavelet decomposition where the decomposition is applied to all frequency channels, but this scheme does not achieve good energy compaction since it sacrifices some spatial localisation properties. Another flexible structure of wavelet decomposition is to apply the two-scale decomposition only to channels that contain a significant amount of information and further decomposition could achieve a better energy compaction. This kind of transform is called wavelet packet (WP) transform. 
Table (1) Energy weight for the four subbands of a number of still test images after first-level wavelet decomposition.

\begin{tabular}{cllllll}
\hline \multirow{2}{*}{$\begin{array}{c}\text { Subban } \\
\mathrm{d}\end{array}$} & \multicolumn{3}{c}{ Natural images } & \multicolumn{3}{c}{ Medical Images } \\
\cline { 2 - 7 } & Lena & Boat & Camera & Goldhill & Chest (CT) & Head (MR) \\
LL & $94.03 \%$ & $94.27 \%$ & $92.92 \%$ & $91.40 \%$ & $99.22 \%$ & $91.82 \%$ \\
LH & $2.58 \%$ & $2.50 \%$ & $3.11 \%$ & $3.12 \%$ & $0.31 \%$ & $3.92 \%$ \\
HL & $1.95 \%$ & $2.23 \%$ & $2.52 \%$ & $3.50 \%$ & $0.32 \%$ & $3.28 \%$ \\
\hline HH & $1.44 \%$ & $1.00 \%$ & $1.45 \%$ & $1.98 \%$ & $0.15 \%$ & $0.98 \%$ \\
\hline
\end{tabular}

\subsection{ORTHOGONAL WAVELETS}

It is the family of wavelets that generate orthonormal bases of. $L^{2}\left(R^{n}\right)$ The most important to image compression are compactly supported orthogonal wavelets. In DWT, compactly supported wavelets correspond to finite impulse response (FIR) filters. But has a major disadvantage, which is their asymmetry.

This property translates into nonlinear phase in the associated FIR filters that may cause artifacts at the borders of the wavelet subbands. This can be avoided by using linear phase wavelet filters. Defining $W_{j}$ as the orthogonal complement of $V_{j}$ in $V_{j-1}$, i.e.,

$$
W_{j} \perp V_{j} \text { and } W_{j} \oplus V_{j}=V_{j-1}
$$

$W_{j}$ can be seen as the amount of detail added when going from resolution $V_{j}$ to the larger resolution $V_{j-1}$. If we want both symmetry and compact support in wavelets, we are led to biorthogonal wavelets, it is more a general class of wavelet transforms.

\subsection{Biorthogonal Wavelets}

It has been mostly used for their symmetry, it is characterised by two wavelets, the analysis wavelet $v(t)$, and the synthesis one $\bar{v}(t)$ [24]. This is can be represented as follows:

$$
x(t)=\sum_{m=-\infty}^{\infty} \sum_{n=-\infty}^{\infty} c_{m, n} \bar{v}_{m, n}(t)
$$

where 


$$
c_{m, n}=\int_{-\infty}^{\infty} v_{m, n}(t) x(t) d t
$$

and

$$
\begin{aligned}
& v_{m, n}(t)=2^{-m / 2} v\left(2^{-m} t-n\right) \\
& \bar{v}_{m, n}(t)=2^{-m / 2} v\left(2^{-m} t-n\right)
\end{aligned}
$$

However, the $v_{m, n}(t)$ are orthogonal to $\bar{v}_{m, n}(t)$, this means: $<v_{m, n}(t), \bar{v}_{m, n}(t)>\delta_{m, k} \delta_{n, l}$

\section{MULTIRESOLUTION WAVELET ANALYSIS}

This section briefly discusses the theory of multiresolution analysis (MRA). The wavelet decomposition of any function $f(x) \in \ell^{2}\left(\Re^{n}\right)$ is implemented using a family of functions generated by dilating and translating a single wavelet function $\psi: \mathfrak{R} \rightarrow \mathfrak{R}$. The function $\psi(x)$ is called the mother wavelet and the function $\psi_{j, n}(x)=2^{\frac{1}{2}} \psi\left(2^{j} x-n\right)$ from an Orthonormal basis for $\ell^{2}(\Re), \psi(x)$ is often obtained from a companion $\phi(x)$ and is known as the scaling function through the following equations:

$$
\phi(x)=\sqrt{2} \sum_{k} h_{0}(k) \phi(2 x-k)
$$

and

$$
\phi(x)=\sqrt{2} \sum_{k} h_{1}(k) \phi(2 x-k)
$$

Where $\left\{h_{0}(k)\right\}$ and $\left\{h_{1}(k)\right\}$ are pair of discrete quadrature mirror filters (lowpass and highpass) for orthogonal system of the form:

$$
h_{1}(k)=(-1)^{k-1} h_{0}(-k+1)
$$

Wavelet transforms can be understood more easily through the concept of multiresolution signal representation [19].

\section{PYRAMIDS ALGORITHM}

It is image transforms where multiple copies of an image at different resolutions are formed. It consists of an image at the bottom of the pyramid that is the highest or limiting resolution together with copies at lower and lower resolution. 
There are many types of pyramids such as, low pass pyramid, a band pass pyramid and a wavelet pyramid. A low pass pyramid consists of the finest resolution image, followed by a half resolution version, followed by a quarter, and so on., where each version is formed from the previous one by an averaging process. The top of the image is a single pixel that is the average of the entire image. In a band pass pyramid the top of the pyramid is a single pixel as well, and every other level contains detail imagery required to generate an image at a desired resolution level from the previous one. The third type of image pyramid is the most frequently used. This is the wavelet transform and it can be thought of as a type of bandpass pyramid which stores separate detail images for horizontal, vertical and diagonal.

\section{WAVELETS FEATURES FOR IMAGE COMPRESSION}

This is a summary of some features of image compression using Wavelets.

1. Wavelet transform has a good energy compact, it is preserved across the transform, i.e. the sum of squares of the wavelet coefficients is equal the sum of squares of the original image.

2. Wavelets can provide a good compression [4, 19], it can perform better than JPEG, both in terms of SNR and image quality. Thus show no blocking effect unlike JPEG.

3. The entire image is transformed and compressed as a single data object using wavelet transforms, rather than block by block. This allows for uniform distribution of compression error across the entire image and at all scales.

4. The wavelet transform methods have been shown to provide integrity at higher compression rates than other methods where integrity of data is important e.g., medical images and fingerprints, etc.

5. Multiresolution properties allow for progressive transmission and zooming, without extra storage.

6. It is a fast performance operation, in addition to symmetry: both the forward and inverse transform have the same complexity, in both compression and decompression phases.

7. Many image operations such as noise reduction and image scaling can be performed on wavelet transformed images.

Resultant wavelet multiresolutions are scaled subbands. Coarse approximation scaled from the original image and the others are detail coefficients where there are a statistical dependence across scale in these coefficients. Efficient encoding is to exploit such dependence. This is how the art wavelet transforms compression is achieved. 


\section{MODIFIED LBG ALGORITHM USING PARTIAL SEARCH PARTIAL DISTORTION}

In basic Vector Quantization (VQ), a full search technique is used, where the Euclidean distance measure is calculated for the entire code vector in the codebook. It results in complexity in computation and time consummation. These are the most serious problems facing VQ. In this paper, the LBG algorithm is modified to make it more efficient in speeding up codebook generation and encoding phase. The improvement is based on the fact that two equal-sized image blocks cannot be closely matched unless their local means and variance are closely matched.

In the proposed modification a significant feature, the combination of variance $\sigma^{2}$ and mean $m$ is used to reject a large number of code vectors from the search consideration without calculating their distortion distance from the training vectors. Then, the partial search is used to find the closed or best match code vectors from the remaining possible match of the codebook.

The algorithm is described as follows:

Step (1). Initialization.

Let $n=$ length of training vectors, $N=$ Codebook size, $k=$ Vector dimension, $C_{0}=$ Initial codebook, $D_{-1}=$ Initial average distortion .

Step (2). Compute the variance $\sigma^{2}$ and mean $m$ values of each code vector in the codebook to find $h=\frac{\sigma^{2}}{m}$, and sort the codebook in ascending order according to the increase of $h$.

Step (3). Calculate the minimum distortion partition. All training vectors are grouped into clusters $S_{i}$ using the minimum distortion rules as follows:

(a). Compute $h_{x}$ for the input vector $x$ (e.g. $\left.h_{x}=\frac{\sigma_{x}^{2}}{m_{x}}\right)$.

(b). Find the best match of $h_{x}$ from the sorted codebook in step (2).

(c). Define the partial codebook, the upper and lower bound from the sorted codebook with a window size $\pm W$ from the best match vector.

(d). Find the best match code vector of the input vector from the partial codebook by calculating the distortion of each codevector and selecting the minimum distortion.

(e ). Repeat ( a ) to ( d ) for all training vector. 
Step (4). Calculate the average distortion $D_{\text {iteration }}$ for this iteration as follows:

$$
D_{\text {iteration }}=\frac{1}{n} \sum_{i=0}^{n-1} \min _{y \in C_{\text {ieration }}} d\left(x_{i}, y\right)
$$

Step (5). If $\left(D_{\text {ittration-1 }}-D_{\text {iteration }}\right) / D_{\text {iteration }} \leq \zeta$, stop with codebook, otherwise go to step (6).

Step (6). Compute the centroid of each cluster and then go to step (1) for next iteration.

\section{SIMULATION RESULTS}

Experiments are performed on standard 256x256 greyscale Lena, Cameraman, Boat and Goldhill images to test the proposed algorithms at several bit rates. The Daubechies filters are used in the experiments with 4-level wavelet decomposition. The lowest band is coded separately from the remaining bands. The results of this algorithm on the above test images are presented. All the images have a mixture of large smooth regions and long oscillatory patterns. In order to evaluate the performance of the algorithm, it is compared to the direct VQ and the standard JPEG. The performance of the algorithm is reported in Fig. 8, Fig. 9 and Fig. 10. Figure 8 shows the Peak Signal to Noise Ratio (PSNR) versus compression ratio for the test images using this algorithm. Figure 9 shows reconstructed 'Cameraman' test image with different compression ratios and PSNR in decibel. As it may be seen, no blocking effect can be noticed and the image quality is acceptable. The images out side the training have less PSNR of approximately $1-1.5 \mathrm{~dB}$.

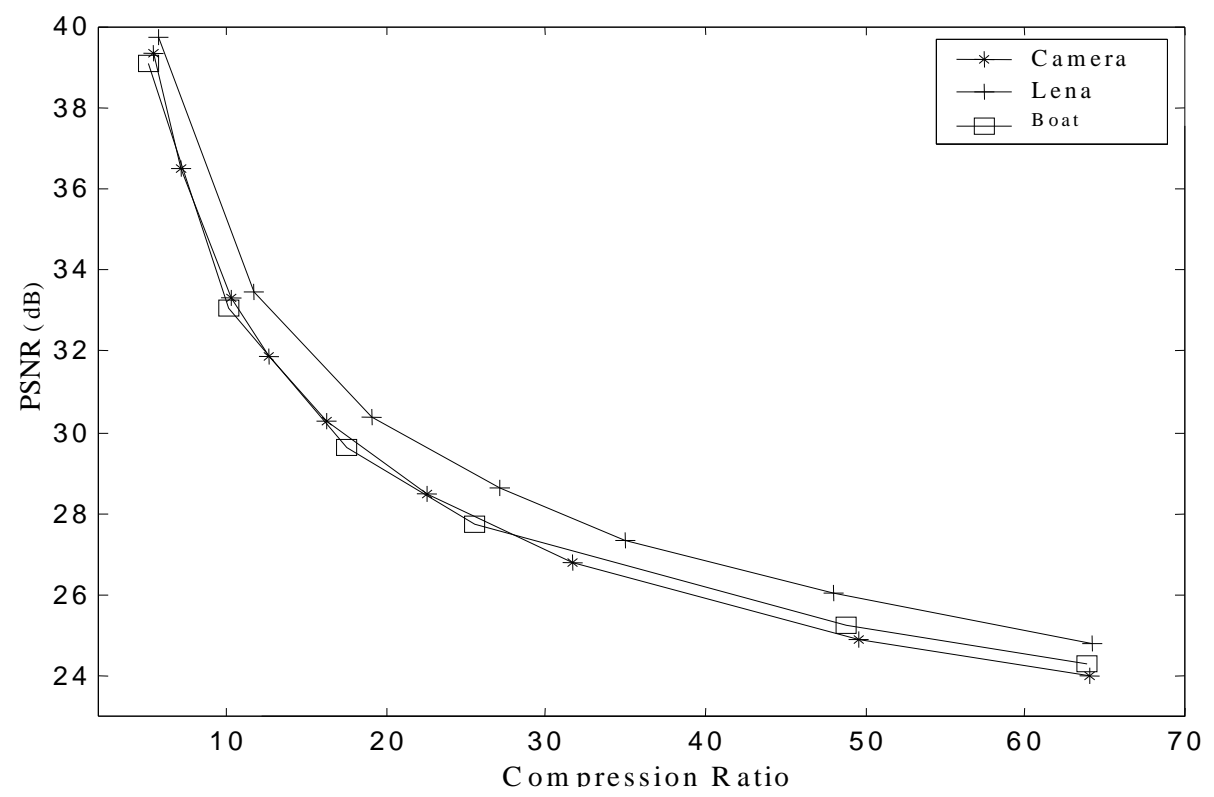

Fig. 8: Compression ratio vs. PSNR of the proposed algorithm upon some of the test images. 


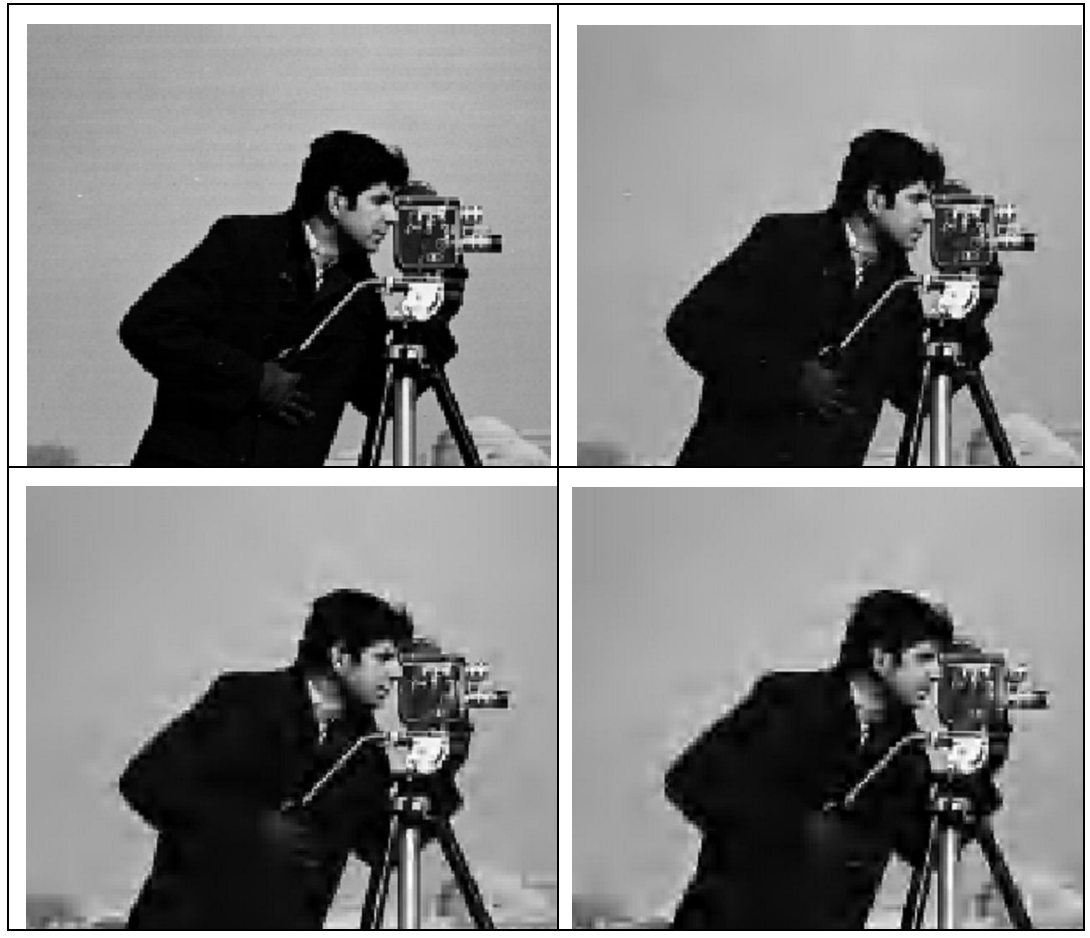

Fig. 9: Simulation results using wavelet transform. (a) Original test image (top left). (b) Compression ratio 12:1, PSNR=32.85 (top right). (c) Compression ratio 31.6:1, PSNR=26.78 (bottom left). (d) Compression ratio 64.25:1, PSNR=23.99 (bottom right).

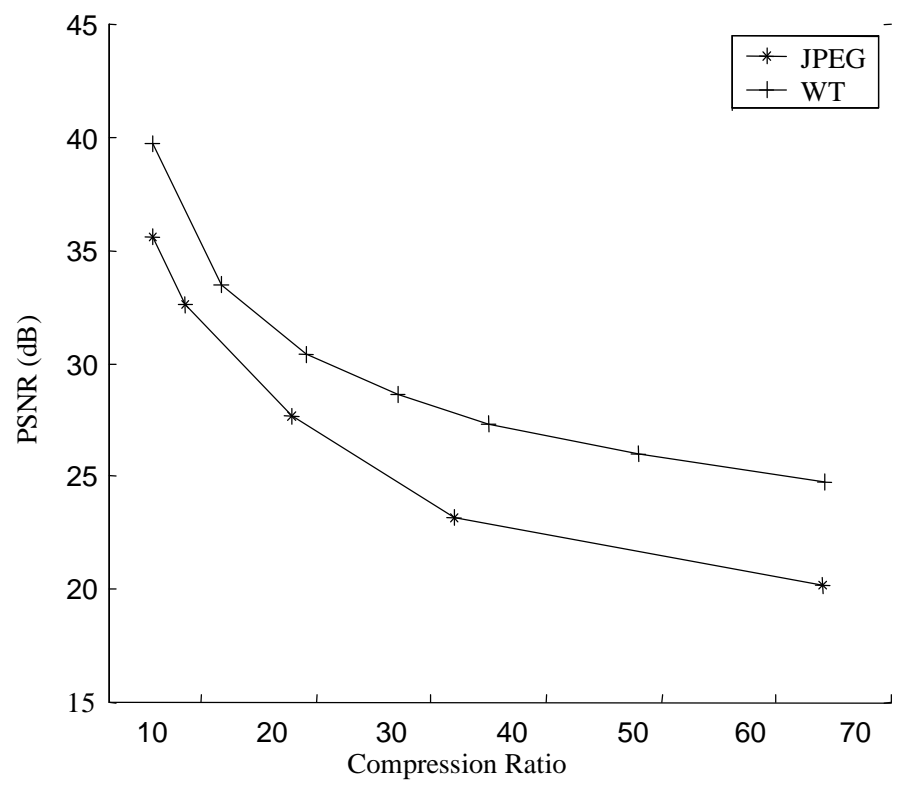

Fig. 10: PSNR Comparison between JPEG and proposed wavelet technique for Cameraman test image. 


\section{COMPARISON WITH OTHER CODERS}

The measure criterion for comparison was PSNR, which can be calculated directly from the original and reconstructed data. Figure 10 shows a comparison of JPEG and the proposed wavelet codec for the test image Cameraman and with a size $256 \times 256$. In terms of statistical error, wavelet codec gives higher signal to noise ratio in two of the examples, Lena and Cameraman. Although all images contained noise introduced by the digitisation process, the wavelet codec effectively removed this noise whilst JPEG spent valuable bits sending this data. As a conclusion, wavelet codec provides a very efficient implementation in terms of execution time, quality and compression ratio. To summarise, the proposed codec perform satisfactory compared to the industrial standard JPEG algorithm as shown in Fig. 11 and much better than vector quantisation technique. These results show that the algorithm provides a highly competitive solution to the problem of image data compression

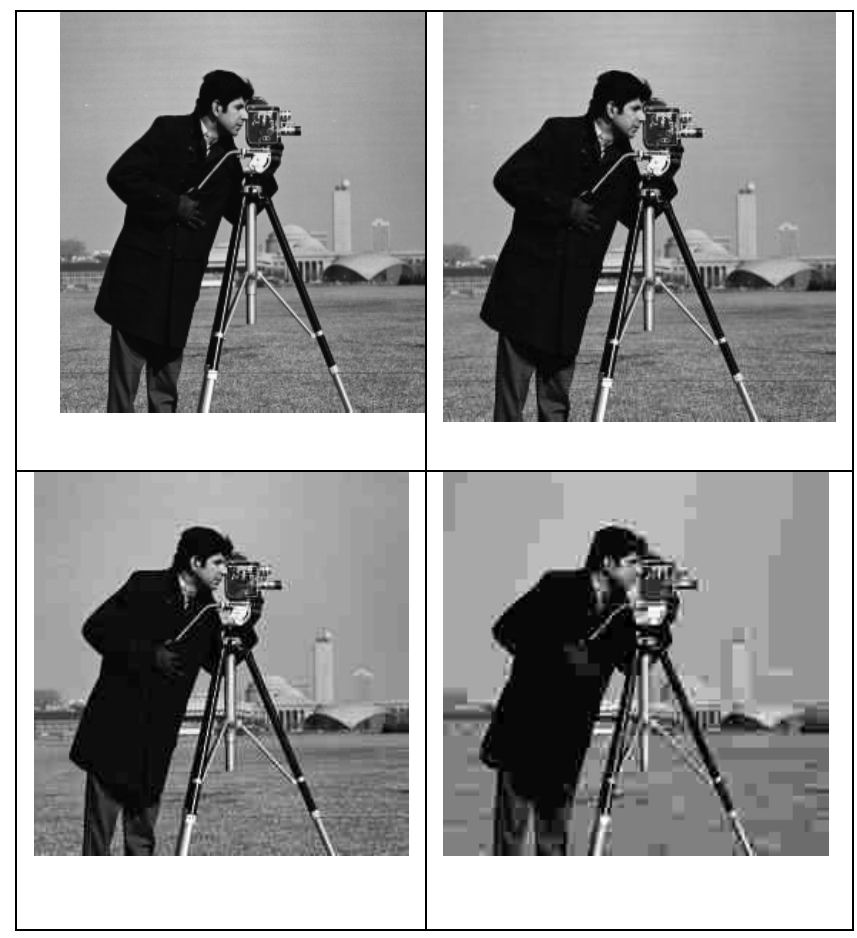

Fig. 11: Simulation results using Standard JPEG. (a) Original test image (top left). (b) Compression ratio 8:1, PSNR=33.19 dB (top right). (c) Compression ratio 32:1, $\mathrm{PSNR}=24.3 \mathrm{~dB}$ (bottom left). (d) Compression ratio 64:1, PSNR=16.9 dB (bottom right).

\section{CONCLUSION \& RECOMMENDATION FOR FURTHER WORK}

The quality of reconstructed images within the training set yielded a compression ratio of $60-50$ and a PSNR was $36-32 \mathrm{~dB}$, with greatly reduced computation and 
execution time needed for codebook design and encoding phase. It reduces the computation complexity to $O(k f)$ arithmetic operations instead of $O(k N)$ in full search, where $\mathrm{N}$ is the number of codevectors, $\mathrm{k}$ is the vector dimension, and $\mathrm{f}$ is the window width, (where $f \ll N$ ). The proposed scheme can save $70-80 \%$ of the VQ encoding time compared to full search VQ. To increase the efficiency, fast algorithm for wavelet transform has to be incorporated. Developing Fast hardware architecture for wavelet transform and vector quantization is an area to be future investigated.

\section{REFERENCES}

[1] [1]. Rioul, O. and Vetterli, M., 'Wavelet and signal Processing', IEEE SP Magazine, October 1991, pp. $14-37$.

[2][2]. Rioul O. and Duhamel P., 'Fast Algorithms for Discrete and Continuous Wavelet Trnasforms', IEEE Trans. On Information Theory, Vol. 38, No. 2, March 1992, pp. 569586.

[3][3]. S. G. Mallat, A Theory for Multiresolution Signal decomposition: The wavelet representation,IEEE Trans. On Pattern Analysis and Machine Intelligence, Vol. 11, NO. 7, pp. $674-693$, July 1989.

[4][4]. DeVore R. A., Jawrth B. and Lucie B. J., 'Image Compression through wavelet Transform', IEEE Trans. On Information Theory, 38, 1991, pp. 719-746.

[5] [5]. Kim Y. H. and Modestino J. W., 'Adaptive Entropy Coded Subband Coding of Images', IEEE Trans. On Image Processing, Vol. 1, January 1992, pp. 31-48.

[6] [6]. Daubechies, The Wavelet Transform, Time-Frequency Localization and Signal Analysis, IEEE Trans. On Information Theory, Vol. 36, No. 5, pp. 961-1004, September 1990.

[7] [7]. Wong, S., Zaremba, G. D. and Huary. HK, 'Radiologic image compression - A review', IEEE Proc., Vol. 83, 1995, pp. $194-219$.

[8][8]. Woods J. W. and O'Neil S., 'Subband Coding of Images', IEEE Trans. Acoustics, Speech, and Signal Processing, Vol. 34, 10, October 1986, pp. 1278-1288.

[9] [9]. Beylkin G., Coifman R., and Rokhlin V., ' Fast wavelet transforms and numerical algorithms I', Comm. Pure and Applied Math., Vol. 54, pp. 141-183, 1991.

[10] [10]. Donoho D. L., ' Nonlinear wavelet methods for recovery of signals, densities and spectra from indirect and noisy data', Technical report, Derpartment of Statistics, Stanford University, Stanford CA, 1994.

[11] [11]. Starck J., Bijaoui A., Lopez B., and Perrier C., 'Image reconstruction by the wavelet transform applied to aperture synthesis', Astronom. and Astrophys., February 1994.

[12] [12]. Hopper T. and Preston F., 'Compression of Gray-scale Fingerprint Images', in Proc. DCC'92, 1992 Data Compression Conf., Snowbird, Utah, March 1992, pp. 309-318.

[13] [13]. Woods J. W., 'Subband Image Coding', Kluwer Academic, 1991.

[14] [14]. Z. Xiong, K. Ramchandran and M. T. Orchard, Space-frequency quantization for wavelet image coding, IEEE Trans. On Image Processing, Vol. 6, No. 5, pp. 677-693, 1997.

[15] [15]. S. G. Mallat, Multifrequency Channel Decompositions of Images and Wavelet Models, IEEE Trans. Acoust., Speech, Signal Processing, Vol. 37, No. 12, Dec. 1989, pp. 2091-2110. 
[16] [16]. Burt P. J. and Adelson E. H., 'The laplacian Pyramid as a Compact Image Code', IEEE Trans. On Communication, Vol. 31, 1983, pp. 532-540.

[17] [17]. J. D. Villasenor, B. Belzer and J. Liao, Wavelet Filter Evaluation for Image Compression, IEEE Trans. On Image Processing, August 1995.

[18] [18]. Daubechies I., 'Ten Lectures on Wavelets', Society for Industrial and Applied Mathematics, Philadelphia, 1992.

[19] [19]. M. Antonini , M. Barlaud and I. Daubechies., Image Coding using Wavelet Transform, IEEE Trans. On Image Processing Vol.1, No.2, pp. 205 - 220, APRIL 1992.

[20] [20]. Vitterli M. and Hereley C., 'Wavelets and filter banks: Theory and design', IEEE Trans. On Signal Processing, Vol. 40, 1992, pp. 2207-2232.

[21] [21]. Wannamaker R. A., 'Fractal Wavelet Compression of Audio Signals', Journal of Audio Engineering Society, Vol. 45, Nos. 7-8, 1997, pp. 540-553.

[22] [22].Hilton H., Jawerth B., and Sengupta A., 'Compressing still and moving images with wavelets', Multimedia Systems, Vol. 2, No. 3, 1994.

[23] [23]. .Khalifa O. O. and Dlay S. S., 'Wavelets for Image Data Compression using bit allocation with Scalar Quantization', S.F., ISIE'97, Guimaraes, Portugal, July 7-11, 1997, pp.77-81.

[24] [24]. O. O. Khalifa and S. S. Dlay, Medical Image Lossless Compression for transmission of cross-limited bandwidth channels, SPIE, Medical Imaging, San Diego, USA, pp. 342346, February 1999.

\section{BIOGRAPHY}

Othman Omran Khalifa received his Bachelor's degree in Electronic Engineering from the Garyounis University, Libya in 1986. He obtained his Master degree in Electronics Science Engineering and PhD in Digital Image Processing from Newcastle University, UK in 1996 and 2000 respectively. He worked in industrial for eight years and he is currently an Assistant Professor at the department of Electrical and Computer Engineering, International Islamic University Malaysia. His area of research interest is Information theory and Coding, Digital image / video Communication, coding and Compression, Wavelets, Fractal and Pattern Recognition. 